\title{
Pacientes com traumatismo cranioencefálico tratados cirurgicamente no serviço de neurocirurgia do Hospital de Base do Distrito Federal (Brasília-Brasil)
}

\author{
Cléciton Braga Tavares', Emerson Brandão Sousa1, Igor Brenno Campbell Borges²,
} Francisca das Chagas Sheyla Almeida Gomes Braga ${ }^{3}$

Unidade de Neurocirurgia do Hospital de Base do Distrito Federal, Brasília, DF, Brasil. Universidade Estadual do PiauI (UESPI), Teresina, PI, Brasil.

\section{RESUMO}

Objetivo: O traumatismo cranioencefálico (TCE) é uma agressão ao encéfalo causada por uma força física externa. No Brasil, é a principal causa de morte de crianças acima de 5 anos de idade e adolescentes. Apresentamos as características epidemiológicas de 194 pacientes com TCE operados no serviço de neurocirurgia do Hospital de Base do Distrito Federal. Métodos: Trata-se de um estudo descritivo, tipo corte transversal, baseado na revisão de prontuários médicos de pacientes com TCE tratados cirurgicamente no período de julho de 2007 a julho de 2012. Resultados: Predomínio do sexo masculino (82,99\%); a faixa etária mais comum é de 21-40 anos (67); a maioria apresentava TCE grave (108) e o principal mecanismo do trauma foi agressão física (57) seguida por queda da própria altura (49). Hematoma subdural crônico (63), hematoma extradural agudo (49) e fratura com afundamento (38) foram os principais achados tomográficos. Há uma relação entre hematoma subdural crônico $(p<0,05 / O R=1,272 / / C 95 \%$ : $1,163-1,391)$, hematoma subdural agudo $(p=0,008 / O R=3,271 / I C 95 \%: 1,309-8,172)$ e atropelamento $(p<0,05 / O R=8,804 / I C$ 95\%: 2,203-35,185) com TCE grave. Conclusão: $A$ maioria dos pacientes era do sexo masculino, na faixa etária entre 21-40 anos, vítima de agressão física e admitida com TCE grave. Há relação entre atropelamento, hematoma subdural agudo e crônico com a gravidade do TCE.

\section{PALAVRAS-CHAVE}

Traumatismos craniocerebrais, perfil de saúde, epidemiologia.

\section{ABSTRACT}

Patients with brain injury surgically treated in neurosurgery service at Hospital de Base do Distrito Federal (Brasilia, Brazil)

Objective: Traumatic brain injury is an injury to the brain caused by an external physical force. In Brazil, it is the leading cause of death in children over 5 years old and teenagers. We present the epidemiological characteristics of 194 patients with traumatic brain injury treated at the Serviço de Neurocirurgia do Hospital de Base do Distrito Federal. Methods: This is a descriptive, cross-sectional type, based on review of medical records of patients with traumatic brain injury treated surgically in the period July 2007 to July 2012. Results: There was a predominance of males (82.99\%), the most common age group is 21-40 years (67), the majority had severe traumatic brain injury (108), the main mechanism of trauma was physical assault (57) and followed by fall from height (49). Chronic subdural hematoma (63), acute epidural hematoma (49) and fracture dip (38) were the main CT findings. There is a relationship between chronic subdural hematoma $(p<0.05 / O R=1,272 / 95 \% \mathrm{Cl}: 1.163$ to 1.391$)$, acute subdural $(p=0.008$ / OR $=3,271 / 95 \%$ Cl: 1.309 to 8.172) and trampling ( $p<0.05 / O R=8,804 / 95 \%$ Cl: 2.203 to 35.185$)$ with severe traumatic brain injury. Conclusion: Most patients were male, age range between 21-40 years, victims of physical assault and admitted with severe traumatic brain injury. There is a relationship between trampling, acute and chronic subdural hematoma with the severity of traumatic brain injury.

\section{KEYWORDS}

Craniocerebral trauma, health profile, epidemiology.

\footnotetext{
1 Neurocirurgião preceptor da Disciplina de Neurologia/Neurocirurgia da Universidade Estadual do Piauí (UESPI), Teresina, PI, Brasil.
}

2 Neurocirurgião do Hospital de Base do Distrito Federal, Brasília, DF, Brasil.

3 Enfermeira do Hospital Universitário do Piauí, Universidade Federal do Piauí (UFPI), Teresina, PI, Brasil. 


\section{Introdução}

O traumatismo cranioencefálico (TCE) é uma agressão ao encéfalo causada por uma força física externa, que acarreta lesão anatômica e/ou comprometimento funcional do couro cabeludo, crânio, meninges ou encéfalo. $^{1,2}$

A lesão definitiva que se estabelece após o TCE é o resultado de mecanismos fisiopatológicos que se iniciam com o acidente e se estendem por dias a semanas. Assim, do ponto de vista didático, as lesões cerebrais são classificadas em primárias e secundárias. ${ }^{3,4}$ As lesões primárias são aquelas que ocorrem no momento do trauma, enquanto as lesões secundárias decorrem de agressões que se iniciam após o momento do acidente, resultantes da interação de fatores intra e extracerebrais., ${ }^{3,4}$

Entre os traumas mecânicos, o TCE é o principal determinante de óbito e sequelas em politraumatizados, sendo, por isso, definido pela Organização Mundial de Saúde como um problema de saúde pública. ${ }^{5,6}$

A incidência geral do TCE nos Estados Unidos foi estimada em 538,2/100.000 habitantes; ${ }^{7}$ taxas um pouco menores são relatadas na Europa (235/100 mil) e Austrália (322/100mil). ${ }^{8,9}$ No Brasil, é a principal causa de morte em crianças acima de 5 anos de idade e responsável por mais de $50 \%$ dos óbitos na adolescência. ${ }^{1}$

Apresentamos neste trabalho as características epidemiológicas de 194 pacientes com TCE tratados cirurgicamente no serviço de neurocirurgia do Hospital de Base do Distrito Federal (HBDF). Os dados levantados são importantes do ponto de vista de saúde pública e gestão de recursos.

\section{Material e métodos}

Trata-se de um estudo descritivo, tipo corte transversal, baseado na revisão de prontuários médicos, de pacientes com TCE tratados cirurgicamente no serviço de neurocirurgia do Hospital de Base do Distrito Federal, Brasília, Brasil, no período de julho de 2007 a julho de 2012.

Os dados levantados foram: faixa etária, sexo, mecanismo de trauma, tipo de lesão cranioencefálica (patologia) e a Escala de Coma de Glasgow à admissão hospitalar. Os prontuários que não apresentavam todos esses dados, no relatório de alta hospitalar, foram excluídos do trabalho.

Os pacientes foram agrupados quanto à idade, em cinco faixas etárias: 0-10 anos, 11-20 anos, 21-40 anos, 41-60 anos e acima de 60 anos. Os mecanismos do trauma foram divididos em onze grupos: acidentes automobilísticos, atropelamento, acidentes motociclísti- cos, queda de altura, queda da própria altura, lesão por arma de fogo, acidentes náuticos, acidentes ciclísticos, acidente durante atividades esportivas, agressão física e queda de material pesado sobre o crânio.

A classificação de gravidade do trauma foi baseada na pontuação da Escala de Coma de Glasgow. ${ }^{10}$ Considerando-se TCE grave: 3-8 pontos, TCE moderado: 9-13 e TCE leve: $14-15{ }^{11}$

As informações foram compiladas em uma planilha do programa Excel-Windows, perfazendo um total de 194 indivíduos $(\mathrm{n}=194)$. Todos os dados foram analisados pelo software Statistical Package for the Social Sciences (SPSS) 20.0.0. As associações foram avaliadas com teste do qui-quadrado, odds ratio e com o teste $\mathrm{T}$ para comparação de médias aritméticas. Um valor de $\mathrm{p}<0,05$ foi considerado estatisticamente significativo.

\section{Resultados}

A maioria dos pacientes foi do sexo masculino (82,99\%), com predomínio da faixa etária de 21-40 anos de idade. A média de idade entre as mulheres (48,58 anos) foi maior que a dos homens (43,23 anos), no entanto a diferença não foi estatisticamente significativa, segundo o teste T para comparação entre médias aritméticas (Gráficos 1, 2 e 3).

Observou-se no gráfico 4 que a maior parte dos indivíduos tratados cirurgicamente apresentou TCE grave à admissão hospitalar, de acordo com a Escala de Coma de Glasgow.

Conforme apresentado no gráfico 5 , as patologias mais operadas foram o hematoma subdural crônico, hematoma extradural agudo e fratura com afundamento.

As agressões físicas e as quedas da própria altura foram os principais mecanismos do trauma (Gráfico 6).

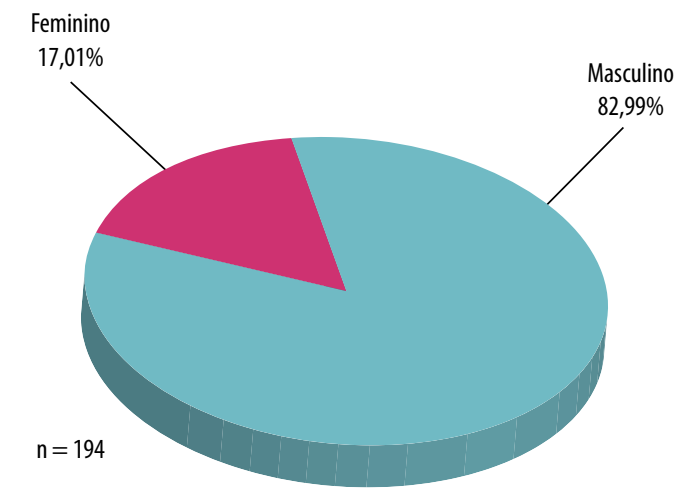

Fonte: Serviço de Arquivo Médico do Hospital de Base do Distrito Federal (Brasília/Brasil).

Figura 1 - Distribuição segundo o sexo dos pacientes com traumatismo cranioencefálico tratados cirurgicamente no serviço de neurocirurgia do HBDF entre julho de 2007 e julho de 2012. 


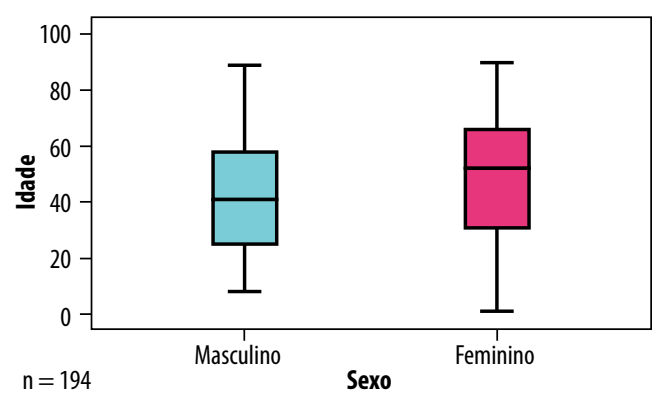

Fonte: Serviço de Arquivo Médico do Hospital de Base do Distrito Federal (Brasília/Brasil).

Idade média: geral 44,14 anos (1-90 anos)

Masculino: 43,23 anos; Feminino: 48,58 anos ( $\mathrm{p}=0,175$, Teste T) Gráfico 2 - Distribuição segundo o sexo e a idade dos pacientes com traumatismo cranioencefálico tratados cirurgicamente no serviço de neurocirurgia do HBDF entre julho de 2007 e julho de 2012.

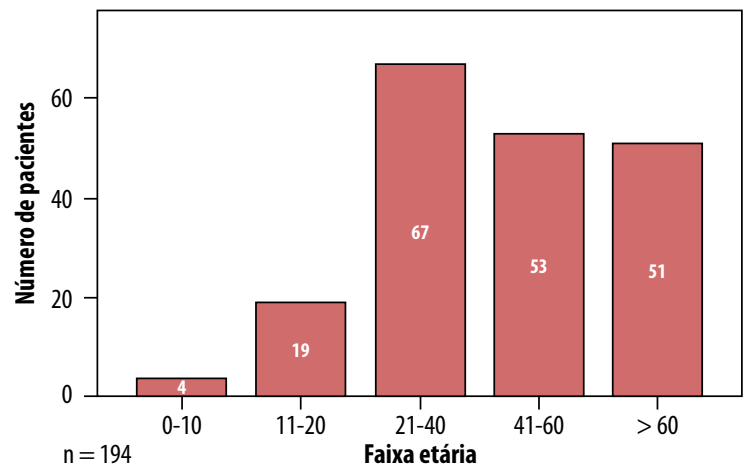

Fonte: Serviço de Arquivo Médico do Hospital de Base do Distrito Federal (Brasília/Brasil).

Gráfico 3 - Distribuição segundo a faixa etária dos pacientes com traumatismo cranioencefálico tratados cirurgicamente no servico de neurocirurgia do HBDF entre julho de 2007 e julho de 2012.

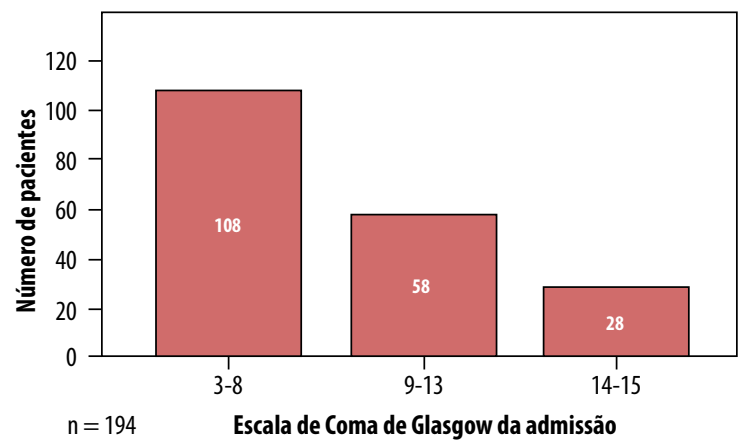

Fonte: Serviço de Arquivo Médico do Hospital de Base do Distrito Federal (Brasília/Brasil).

Gráfico 4 - Distribuição segundo a Escala de Coma de Glasgow dos pacientes com traumatismo cranioencefálico tratados cirurgicamente no serviço de neurocirurgia do HBDF entre julho de 2007 e julho de 2012.

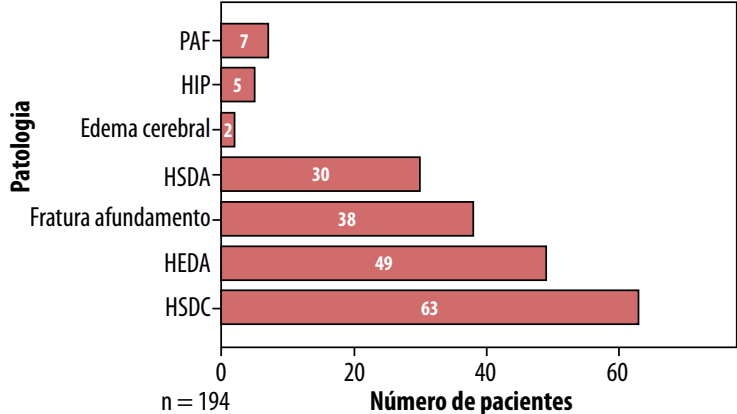

Fonte: Serviço de Arquivo Médico do Hospital de Base do Distrito Federa (Brasília/Brasil)

Referências: HSDC: hematoma subdural crônico; HEDA: hematoma extradural agudo; HSDA: hematoma subdural agudo; HIP: hematoma intraparenquimatoso; PAF: perfuração por arma de fogo.

Gráfico 5 - Distribuição segundo a patologia dos pacientes com traumatismo cranioencefálico tratados cirurgicamente no serviço de neurocirurgia do HBDF entre julho de 2007 e julho de 2012.

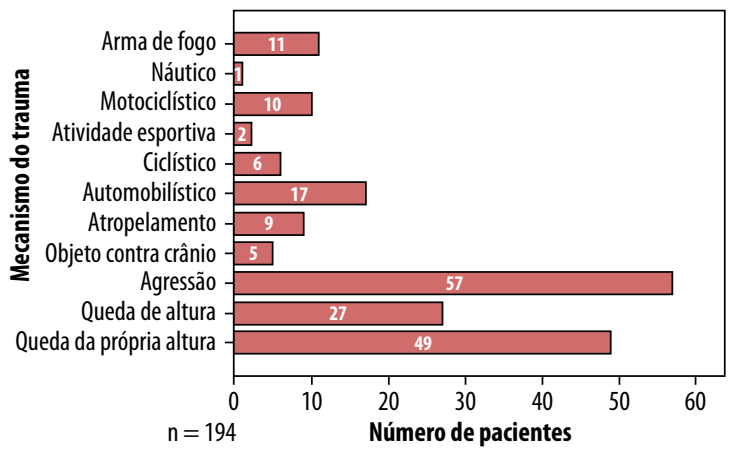

Fonte: Serviço de Arquivo Médico do Hospital de Base do Distrito Federal (Brasília/Brasil).

Gráfico 6 - Distribuição segundo o mecanismo do trauma

dos pacientes com traumatismo cranioencefálico tratados cirurgicamente no serviço de neurocirurgia do HBDF entre julho de 2007 e julho de 2012.

Não há relação entre sexo e faixa etária com a gravidade do trauma, uma vez que as diferenças observadas nas tabelas 1 e 2 não foram estatisticamente significativas.

Existe relação entre as patologias/achados tomográficos, especialmente o hematoma subdural crônico e o hematoma subdural agudo, com o TCE moderado e grave, uma vez que o $p$ é estatisticamente significativo (Tabelas 3, 4 e 5).

Foi observada relação entre atropelamento e gravidade do TCE quando comparado aos outros mecanismos de trauma (Tabelas 6 e 7).

\section{Discussão}

Houve predomínio do sexo masculino e da faixa etária de 21-40 anos de idade. Dados condizentes com a literatura vigente e explicados pelo fato de os homens 
jovens se exporem mais às atividades laborativas e recreativas de risco e serem os principais envolvidos em comportamentos violentos. ${ }^{12-16}$ A média de idade foi elevada, talvez devido ao grande número de pacientes com hematomas subdurais crônicos, uma patologia mais comum em indivíduos de maior faixa etária. ${ }^{1,16}$

A maioria dos indivíduos foi admitida com TCE grave. Dados semelhantes aos publicados por Ruy e Rosa, ${ }^{3}$ que avaliaram vítimas de TCE admitidas na unidade de terapia intensiva. Teoricamente, tanto os pacientes admitidos em terapia intensiva quanto os operados são mais graves e possuem menor Escala de Coma de Glas- gow. No entanto, quando avaliamos todos os pacientes admitidos no pronto-socorro, há predomínio do TCE leve na literatura. ${ }^{1,5,17,18}$

As patologias mais operadas foram hematoma subdural crônico, hematoma extradural agudo e fratura com afundamento; achados variáveis na literatura. Segundo Maia et al., ${ }^{1}$ as patologias mais encontradas, não necessariamente cirúrgicas, foram as contusões, hemorragia subaracnóidea traumática e hematoma subdural agudo. Segundo Moura et al., ${ }^{17}$ contusão, hematoma extradural agudo e hemorragia subaracnóidea traumática foram os principais achados tomográficos.

\begin{tabular}{|c|c|c|c|c|c|}
\hline \multicolumn{6}{|c|}{$\begin{array}{l}\text { Tabela } 1 \text { - Relação entre a gravidade do trauma e o sexo dos pacientes com traumatismo cranioencefálico } \\
\text { tratados cirurgicamente no serviço de neurocirurgia do HBDF entre julho de } 2007 \text { e julho de } 2012\end{array}$} \\
\hline & & & \multicolumn{2}{|c|}{ Gravidade } & \multirow[t]{2}{*}{ Total } \\
\hline & & & Leve & Moderado/Grave & \\
\hline \multirow[t]{6}{*}{ Sexo } & Masculino & Absoluto & 23 & 138 & 161 \\
\hline & & Porcentagem & $14,3 \%$ & $85,7 \%$ & $100 \%$ \\
\hline & & Residual ajustado & $-0,1$ & 0,1 & \\
\hline & Feminino & Absoluto & 5 & 28 & 33 \\
\hline & & Porcentagem & $15,2 \%$ & $84,8 \%$ & $100 \%$ \\
\hline & & Residual ajustado & 0,1 & $-0,1$ & \\
\hline \multirow[t]{2}{*}{ Total } & & Absoluto & 28 & 166 & 194 \\
\hline & & Porcentagem & $14,4 \%$ & $85,6 \%$ & $100 \%$ \\
\hline
\end{tabular}

$\mathrm{n}=194 ; \mathrm{X}^{2}=0,017 ; \mathrm{p}=0,897 ;$ OR $=0,933 ;$ IC 95\% $(0,327-2,664)$.

Fonte: Serviço de Arquivo Médico do Hospital de Base do Distrito Federal (Brasília/Brasil).

\begin{tabular}{|c|c|c|c|c|c|}
\hline & & & \multicolumn{2}{|c|}{ Gravidade } & \multirow[t]{2}{*}{ Total } \\
\hline & & & Leve & Moderado/Grave & \\
\hline \multirow[t]{15}{*}{ Faixa etária } & $0-10$ & Absoluto & 1 & 3 & 4 \\
\hline & & Porcentagem & $25 \%$ & $75 \%$ & $100 \%$ \\
\hline & & Residual ajustado & 0,6 & $-0,2$ & \\
\hline & $11-20$ & Absoluto & 5 & 14 & 19 \\
\hline & & Porcentagem & $26,3 \%$ & $73,7 \%$ & $100 \%$ \\
\hline & & Residual ajustado & 1,4 & $-0,6$ & \\
\hline & $21-40$ & Absoluto & 11 & 56 & 67 \\
\hline & & Porcentagem & $16,4 \%$ & $83,6 \%$ & $100 \%$ \\
\hline & & Residual ajustado & 0,4 & $-0,2$ & \\
\hline & $41-60$ & Absoluto & 9 & 44 & 53 \\
\hline & & Porcentagem & $17 \%$ & $83 \%$ & $100 \%$ \\
\hline & & Residual ajustado & 0,5 & $-0,2$ & \\
\hline & $>60$ & Absoluto & 2 & 49 & 51 \\
\hline & & Porcentagem & $3,9 \%$ & $96,1 \%$ & $100 \%$ \\
\hline & & Residual ajustado & $-2,0$ & 0,8 & \\
\hline \multirow[t]{2}{*}{ Total } & & Absoluto & 28 & 166 & 194 \\
\hline & & Porcentagem & $14,4 \%$ & $85,6 \%$ & $100 \%$ \\
\hline
\end{tabular}

$\mathrm{n}=194 ; \mathrm{X}^{2}=7,589 ; \mathrm{p}=0,108$.

Fonte: Serviço de Arquivo Médico do Hospital de Base do Distrito Federal (Brasília/Brasil). 


\begin{tabular}{|c|c|c|c|c|c|}
\hline \multicolumn{6}{|c|}{$\begin{array}{c}\text { Tabela } 3 \text { - Relação entre a gravidade do trauma e a patologia dos pacientes com traumatismo cranioencefálico } \\
\text { tratados cirurgicamente no serviço de neurocirurgia do HBDF entre julho de } 2007 \text { e julho de } 2012\end{array}$} \\
\hline & & & \multicolumn{2}{|c|}{ Gravidade } & \multirow[t]{2}{*}{ Total } \\
\hline & & & Leve & Moderada/Grave & \\
\hline \multirow[t]{21}{*}{ Patologia } & HSDC & Absoluto & 0 & 63 & 63 \\
\hline & & Porcentagem & $0 \%$ & $100 \%$ & $100 \%$ \\
\hline & & Residual ajustado & $-4,0$ & 4,0 & \\
\hline & HEDA & Absoluto & 11 & 38 & 49 \\
\hline & & Porcentagem & $22,4 \%$ & $77,6 \%$ & $100 \%$ \\
\hline & & Residual ajustado & 1,8 & $-1,8$ & \\
\hline & Afundameto de crânio & Absoluto & 7 & 31 & 38 \\
\hline & & Porcentagem & $18,4 \%$ & $81,6 \%$ & $100 \%$ \\
\hline & & Residual ajustado & 0,8 & $-0,8$ & \\
\hline & HSDA & Absoluto & 9 & 21 & 30 \\
\hline & & Porcentagem & $30 \%$ & $70 \%$ & $100 \%$ \\
\hline & & Residual ajustado & 2,6 & $-2,6$ & \\
\hline & Edema cerebral & Absoluto & 1 & 1 & 2 \\
\hline & & Porcentagem & $50 \%$ & $50 \%$ & $100 \%$ \\
\hline & & Residual ajustado & 1,4 & $-1,4$ & \\
\hline & HIP & Absoluto & 0 & 5 & 5 \\
\hline & & Porcentagem & $0 \%$ & $100 \%$ & $100 \%$ \\
\hline & & Residual ajustado & $-0,9$ & 0,9 & \\
\hline & PAF & Absoluto & 0 & 7 & 7 \\
\hline & & Porcentagem & $0 \%$ & $100 \%$ & $100 \%$ \\
\hline & & Residual ajustado & $-1,1$ & 1,1 & \\
\hline \multirow[t]{2}{*}{ Total } & & Porcentagem absoluto & 28 & 166 & 194 \\
\hline & & & $14,4 \%$ & $85,6 \%$ & $100 \%$ \\
\hline
\end{tabular}

$\mathrm{n}=194 ; \mathrm{X}^{2}=23,625 ; \mathrm{p}=0,001$.

HSDC: hematoma subdural crônico; HEDA: hematoma extradural agudo; HSDA: hematoma subdural agudo; HIP: hematoma intraparenquimatoso; PAF: perfuração por arma de fogo. Fonte: Serviço de Arquivo Médico do Hospital de Base do Distrito Federal (Brasília/Brasil).

\begin{tabular}{|c|c|c|c|c|c|}
\hline \multicolumn{6}{|c|}{$\begin{array}{c}\text { Tabela } 4 \text { - Relação entre a gravidade do trauma e a patologia dos pacientes com traumatismo cranioencefálico } \\
\text { tratados cirurgicamente no serviço de neurocirurgia do HBDF entre julho de } 2007 \text { e julho de } 2012\end{array}$} \\
\hline & & & \multicolumn{2}{|c|}{ Gravidade } & \multirow[t]{2}{*}{ Total } \\
\hline & & & Leve & Moderada/Grave & \\
\hline \multirow[t]{6}{*}{ Patologia } & HSDC & Absoluto & 0 & 63 & 63 \\
\hline & & Porcentagem & $0,0 \%$ & $100 \%$ & $100 \%$ \\
\hline & & Residual ajustado & $-4,0$ & 4,0 & \\
\hline & Outros & Absoluto & 28 & 103 & 131 \\
\hline & & Porcentagem & $21,4 \%$ & 78,65 & $100 \%$ \\
\hline & & Residual ajustado & 4 & $-4,0$ & \\
\hline \multirow[t]{2}{*}{ Total } & & Absoluto & 28 & 166 & 194 \\
\hline & & Porcentagem & $14,4 \%$ & $85,6 \%$ & $100 \%$ \\
\hline
\end{tabular}

$\mathrm{n}=194 ; \mathrm{X}^{2}=15,737 ; \mathrm{p}<0,05 ;$ OR $=1,272 ;$ IC $95 \%(1,163-1,391)$.

HSDC: hematoma subdural crônico.

Fonte: Serviço de Arquivo Médico do Hospital de Base do Distrito Federal (Brasília/Brasil). 


\begin{tabular}{|c|c|c|c|c|c|}
\hline & & & \multicolumn{2}{|c|}{ Gravidade } & \multirow[t]{2}{*}{ Total } \\
\hline & & & Leve & Moderada/Grave & \\
\hline \multirow[t]{6}{*}{ Patologia } & HSDA & Absoluto & 9 & 21 & 30 \\
\hline & & Porcentagem & $30,0 \%$ & $70 \%$ & $100 \%$ \\
\hline & & Residual ajustado & 2,6 & $-2,6$ & \\
\hline & Outros & Absoluto & 19 & 145 & 164 \\
\hline & & Porcentagem & $11,6 \%$ & $88,4 \%$ & $100 \%$ \\
\hline & & Residual ajustado & $-2,6$ & 2,6 & \\
\hline \multirow[t]{2}{*}{ Total } & & Absoluto & 28 & 166 & 194 \\
\hline & & Porcentagem & $14,4 \%$ & $85,6 \%$ & $100 \%$ \\
\hline
\end{tabular}

$\mathrm{n}=194 ; \mathrm{X}^{2}=6,963 ; \mathrm{p}=0,008 ;$ OR $=3,271 ;$ IC $95 \%(1,309-8,172)$.

HSDA: hematoma subdural agudo.

Fonte: Serviço de Arquivo Médico do Hospital de Base do Distrito Federal (Brasília/Brasil).

Tabela 6 - Relação entre a gravidade e o mecanismo do trauma dos pacientes com traumatismo cranioencefálico tratados cirurgicamente no serviço de neurocirurgia do HBDF entre julho de 2007 e julho de 2012

\begin{tabular}{|c|c|c|c|c|c|}
\hline & & & \multicolumn{2}{|c|}{ Gravidade } & \multirow[t]{2}{*}{ Total } \\
\hline & & & Leve & Moderada/Grave & \\
\hline \multirow{21}{*}{ 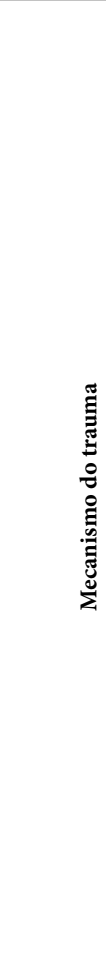 } & \multirow[t]{2}{*}{ Queda da própria altura } & Absoluto & 3 & 46 & 49 \\
\hline & & Residual ajustado & $-1,9$ & 1,9 & $100 \%$ \\
\hline & \multirow[t]{2}{*}{ Queda de altura } & Absoluto & 3 & 24 & 27 \\
\hline & & Residual ajustado & $-0,5$ & 0,5 & $100 \%$ \\
\hline & \multirow[t]{2}{*}{ Agressão } & Absoluto & 8 & 49 & 57 \\
\hline & & Residual ajustado & $-0,1$ & 0,1 & $100 \%$ \\
\hline & \multirow[t]{2}{*}{ Objeto contra o crânio } & Absoluto & 1 & 4 & 5 \\
\hline & & Residual ajustado & 0,4 & $-0,4$ & $100 \%$ \\
\hline & \multirow[t]{2}{*}{ Atropelamento } & Absoluto & 5 & 4 & 9 \\
\hline & & Residual ajustado & 3,6 & $-3,6$ & $100 \%$ \\
\hline & \multirow[t]{2}{*}{ Automobilístico } & Absoluto & 2 & 15 & 17 \\
\hline & & Residual ajustado & $-0,3$ & 0,3 & $100 \%$ \\
\hline & \multirow[t]{2}{*}{ Ciclístico } & Absoluto & 1 & 5 & 6 \\
\hline & & Residual ajustado & 0,2 & $-0,2$ & $100 \%$ \\
\hline & \multirow[t]{2}{*}{ Atividade esportiva } & Absoluto & 1 & 1 & 2 \\
\hline & & Residual ajustado & 1,4 & $-1,4$ & $100 \%$ \\
\hline & \multirow[t]{2}{*}{ Motociclístico } & Absoluto & 3 & 7 & 10 \\
\hline & & Residual ajustado & 1,4 & $-1,4$ & $100 \%$ \\
\hline & \multirow[t]{2}{*}{ Náutico } & Absoluto & 0 & 1 & 1 \\
\hline & & Residual ajustado & $-0,4$ & 0,4 & $100 \%$ \\
\hline & \multirow[t]{4}{*}{$\begin{array}{l}\text { Agressão por } \\
\text { arma de fogo }\end{array}$} & Absoluto & 1 & 10 & 11 \\
\hline & & Residual ajustado & $-0,5$ & 0,5 & $100 \%$ \\
\hline \multirow[t]{2}{*}{ Total } & & Absoluto & 28 & 166 & 194 \\
\hline & & Porcentagem & $14,4 \%$ & $85,6 \%$ & $100 \%$ \\
\hline
\end{tabular}

$\mathrm{n}=194 ; \mathrm{X}^{2}=19,994 ; \mathrm{p}=0,02$.

Fonte: Serviço de Arquivo Médico do Hospital de Base do Distrito Federal (Brasília/Brasil). 
Tabela 7 - Relação entre a gravidade e o mecanismo do trauma dos pacientes com traumatismo cranioencefálico tratados cirurgicamente no serviço de neurocirurgia do HBDF entre julho de 2007 e julho de 2012

\begin{tabular}{|c|c|c|c|c|c|}
\hline & & & \multicolumn{2}{|c|}{ Gravidade } & \multirow[t]{2}{*}{ Total } \\
\hline & & & Leve & Moderada/Grave & \\
\hline \multirow{6}{*}{ 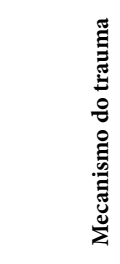 } & Atropelamento & Absoluto & 5 & 4 & 9 \\
\hline & & Porcentagem & $5,6 \%$ & $44,4 \%$ & $100 \%$ \\
\hline & & Residual ajustado & 3,6 & $-3,6$ & \\
\hline & Outros & Absoluto & 23 & 162 & 185 \\
\hline & & Porcentagem & $12,4 \%$ & $87,6 \%$ & $100 \%$ \\
\hline & & Residual ajustado & $-3,6$ & 3,6 & \\
\hline \multirow[t]{2}{*}{ Total } & & Absoluto & 28 & 166 & 194 \\
\hline & & Porcentagem & $14,4 \%$ & $85,6 \%$ & $100 \%$ \\
\hline
\end{tabular}

$\mathrm{n}=194 ; \mathrm{X}^{2}=12,923 ; \mathrm{p}<0,05 ;$ OR $=8,804 ;$ IC $95 \%(2,203-35,185)$.

Fonte: Serviço de Arquivo Médico do Hospital de Base do Distrito Federal (Brasília/Brasil)

Os principais mecanismos do trauma foram as agressões físicas e as quedas da própria altura, o que difere da literatura nacional, que aponta os acidentes de trânsito como a principal causa de traumatismo craniano. ${ }^{17}$ No entanto, como exceção a essa regra e ratificando os nossos achados, podemos citar o trabalho de Melo et al. ${ }^{5}$ que aponta a agressão física como a principal causa de TCE. De modo geral, os mecanismos do trauma dependem das características demográficas, habitacionais e, principalmente, socioeconômicas de cada região.

Não houve relação entre sexo e faixa etária com a gravidade do trauma. Ocorreu relação entre o hematoma subdural crônico e o hematoma subdural agudo com o TCE moderado e grave. As alterações tomográficas são mais frequentes entre os pacientes com TCE moderado e grave, segundo a literatura pesquisada. ${ }^{14,19,20} \mathrm{O}$ hematoma subdural agudo está muito associado a traumas de alto impacto, lesões sistemas e cerebrais, o que aumenta, sobremaneira, sua morbimortalidade. ${ }^{11}$ Como o hematoma subdural crônico ocorre mais frequentemente em indivíduos idosos, a idade avançada também justificaria a maior gravidade do quadro.

Observou-se relação entre atropelamento e gravidade do TCE quando comparado aos outros mecanismos do trauma. Não existem muitos dados na literatura para corroborar esse achado. ${ }^{17}$

\section{Conclusão}

A maioria dos pacientes com TCE tratados cirurgicamente, no serviço de neurocirurgia do Hospital de Base do Distrito Federal, no período de julho de 2007 a julho de 2012, era do sexo masculino, na faixa etária compreendida entre 21-40 anos de idade, vítimas de agressão física e admitidos com TCE grave.
Há relação entre atropelamento, hematoma subdural agudo e crônico com a gravidade do TCE. No entanto, não existe essa mesma relação entre os outros itens pesquisados, como sexo e faixa etária.

\section{Conflito de interesses}

Os autores declaram não haver conflito de interesses.

\section{Referências}

1. Maia BG, Portela LED, Paula FRP, Cotta GD, Cotta MAL, Públio PG, et al. Perfil clínico-epidemiológico das ocorrências de traumatismo cranioencefálico. Rev Neurocienc. 2013;21(1):43-52.

2. David CA. Traumatismo cerebral. In: Jones HR, editor. Neurologia de Netter. Porto Alegre: Artmed; 2006. p. 672-85.

3. Ruy EL, Rosa MI. Perfil epidemiológico de pacientes com traumatismo cranioencefálico. ACM Arq Catarin Med. 2011;4(3):17-20.

4. Goodman JC. Pathophysiology - mild and moderate brain injuries. In: Marion DW, editor. Traumatic brain injury. New York: Thieme; 1999. p. 143-54.

5. Melo JR, Silva RA, Moreira ED Jr. Characteristics of patients with head injury at Salvador City (Bahia-Brazil). Arq Neuropsiquiatr. 2004;62(3A):711-4.

6. IBGE. Instituto Brasileiro de Geografia e Estatística. Resultados do Universo do Censo 2000. Disponível em: $<$ http://www.ibge.gov.br/cidade- sat/xtras/perfil>. Acesso em: 26 mar. 2002.

7. Rutland-Brown W, Langlois JA, Thomas KE, Xi YL. Incidence of traumatic brain injury in the United States, 2003. J Head Trauma Rehabil. 2006;21(6):544-8.

8. Tagliaferri F, Compagnone C, Korsic M, Servadei F, Kraus J. A systematic review of brain injury epidemiology in Europe. Acta Neurochir (Wien). 2006;148(3):255-68.

9. Hillier SL, Hiller JE, Metzer J. Epidemiology of traumatic brain injury in South Australia. Brain Inj. 1997;11(9):649-59. 
10. Teasdale G, Jennett B. Assessment of coma and impaired consciousness practical scale. Lancet. 1974;2(7872):81-4.

11. Greenberg MS. Manual de neurocirurgia. Traumatismo craniano. $7^{\text {a }}$ ed. Porto Alegre: Artmed; 2013. p. 946-1026.

12. Koizumi MS, Lebrão ML, Mello-Jorge MH, Primerano V. Morbidity and mortality due to traumatic brain injury in São Paulo City, Brazil, 1997. Arq Neuropsiquiatr. 2000;58(1):81-9.

13. Bastos YG, Andrade SM, Soares DA. Characteristics of traffic accidents and victims treated through a pre-hospital service in a city in southern Brazil, 1997/2000. Cad Saude Publica. 2005;21(3):815-22.

14. Bruns $J \mathrm{~J}$, Hauser WA. The epidemiology of traumatic brain injury: a review. Epilepsia. 2003;44(Suppl 10):2-10.

15. Ramos SEM, Silva MKB, Siqueira GR, Vieira RAG, França WLC. Aspectos epidemiológicos dos traumatismos cranioencefálicos atendidos no hospital regional do agreste de Pernambuco de 2006 a 2007. RBPS. 2010;23(1):4-10.

16. Sousa EB, Brandão LF, Tavares CB, Borges IB, Neto NG, Kessler IM. Epidemiological characteristics of 778 patients who underwent surgical drainage of chronic subdural hematomas in Brasília, Brazil. BMC Surg. 2013;13:5.

17. Moura JC, Rangel BLR, Creôncio SCE, Pernambuco JRB. Perfil clínico-epidemiológico de traumatismo cranioencefálico do Hospital de Urgências e Traumas no município de Petrolina, estado de Pernambuco. Arq Bras Neurocir. 201130(3):99-104

18. Masini M. Perfil epidemiológico do traumatismo cranioencefálico no Distrito Federal em 1991. J Bras Neurocirurg. 1994;5(2):61-8.

19. Morgado FL, Rossi LA. Correlação entre a escala de coma de Glasgow e os achados de imagem de tomografia computadorizada em pacientes vítimas de traumatismo cranioencefálico. Radiol Bras. 2011;44:35-41.

20. Mota JP, Barja PR. Estudo epidemiológico - Traumatismo cranioencefálico realizado no Hospital Universitário Regional de Maringá. In: IX Encontro Latino-Americano de Iniciação Científica e V Encontro Latino-Americano de Pós-Graduação, Universidade do Vale do Paraíba; 2009. p. 1666-9.

\section{Endereço para correspondência}

Cléciton Braga Tavares

Conjunto IAPEP, bloco C, ap. 32, Ilhotas

64015-040 - Teresina, PI, Brasil

Telefones: (86) 3223-0191/(86) 9925-9772

E-mail: bragatavares@yahoo.com.br 